\title{
Identification and characterization of HAK/ KUP/KT potassium transporter gene family in barley and their expression under abiotic stress
}

Kangfeng Cai ${ }^{1,2}$, Fanrong Zeng ${ }^{2}$, Junmei Wang ${ }^{1}$ and Guoping Zhang ${ }^{2 *}$

\begin{abstract}
Background: HAK/KUP/KT (High-affinity $\mathrm{K}^{+}$transporters $/ \mathrm{K}^{+}$uptake permeases $/ \mathrm{K}^{+}$transporters) is the largest potassium transporter family in plants, and plays pivotal roles in $\mathrm{K}^{+}$uptake and transport, as well as biotic and abiotic stress responses. However, our understanding of the gene family in barley (Hordeum vulgare L.) is quite limited.

Results: In the present study, we identified 27 barley HAK/KUP/KT genes (hereafter called HvHAKs) through a genome-wide analysis. These HvHAKs were unevenly distributed on seven chromosomes, and could be phylogenetically classified into four clusters. All HvHAK protein sequences possessed the conserved motifs and domains. However, the substantial difference existed among HAK members in cis-acting elements and tissue expression patterns. Wheat had the most orthologous genes to barley HAKs, followed by Brachypodium distachyon, rice and maize. In addition, six barley HAK genes were selected to investigate their expression profiling in response to three abiotic stresses by qRT-PCR, and their expression levels were all up-regulated under salt, hyperosmotic and potassium deficiency treatments.
\end{abstract}

Conclusion: Twenty seven HAK genes (HvHAKs) were identified in barley, and they differ in tissue expression patterns and responses to salt stress, drought stress and potassium deficiency.

Keywords: HAK/KUP/KT, Gene family, Barley, Gene expression, Abiotic stress response

\section{Background}

Potassium (K) is the second most abundant mineral nutrient in plants, comprising $2-10 \%$ of plant dry weight [1]. $\mathrm{K}$ is not only essential for plants to maintain normal physiological and biochemical processes such as stomatal movement, photosynthesis, osmoregulation, protein synthesis, enzyme activation, but also involved in the responses to biotic and abiotic stresses [2, 3]. $\mathrm{K}$ in soil exists in several different states/pools $[4,5]$, and available

\footnotetext{
* Correspondence: zhanggp@zju.edu.cn

${ }^{2}$ Institute of Crop Science, Zhejiang University, Hangzhou 310058, China Full list of author information is available at the end of the article
}

$\mathrm{K}$ for plants is commonly quite low due to the strong $\mathrm{K}$ adsorption by 2:1 silicate minerals [4]. In plant cells, the cytosolic $\mathrm{K}^{+}$concentration is relatively constant, ranging from 60 to $200 \mathrm{mM}$, while vacuolar $\mathrm{K}^{+}$concentration is more variable, ranging from $20 \mathrm{mM}$ (in $\mathrm{K}^{+}$-deficient plants) to $500 \mathrm{mM}$ (in specialized guard cells) $[1,6,7]$. In addition, apoplastic $\mathrm{K}^{+}$concentration also shows a large variation, ranging from 10 to $200 \mathrm{mM}$ or even up to $500 \mathrm{mM}$ [8]. In short, $\mathrm{K}$ concentration in plants differs greatly between species, tissues, and cell organs.

As sessile organisms, plants have evolved the efficient $\mathrm{K}^{+}$transport systems to maintain optimal growth under

C C The Author(s). 2021 Open Access This article is licensed under a Creative Commons Attribution 4.0 International License, which permits use, sharing, adaptation, distribution and reproduction in any medium or format, as long as you give appropriate credit to the original author(s) and the source, provide a link to the Creative Commons licence, and indicate if changes were made. The images or other third party material in this article are included in the article's Creative Commons licence, unless indicated otherwise in a credit line to the material. If material is not included in the article's Creative Commons licence and your intended use is not permitted by statutory regulation or exceeds the permitted use, you will need to obtain permission directly from the copyright holder. To view a copy of this licence, visit http://creativecommons.org/licenses/by/4.0/ The Creative Commons Public Domain Dedication waiver (http://creativecommons.org/publicdomain/zero/1.0/) applies to the data made available in this article, unless otherwise stated in a credit line to the data. 
the conditions of highly variable $\mathrm{K}^{+}$levels [9]. In plants, $\mathrm{K}^{+}$is first taken up by roots, and then translocated to aerial parts and distributed within cells into different compartments [10]. All these processes are involved in $\mathrm{K}^{+}$transmembrane transport, which is achieved mainly by $\mathrm{K}^{+}$channels and transporters. So far, $\mathrm{K}^{+}$uptake systems are better identified and characterized in Arabidopsis thaliana than in any other plant species. Currently it is well documented that non-selective cation channels (NSCC) probably play the major role in $\mathrm{K}^{+}$uptake at high concentrations $(>10 \mathrm{mM})$, while the low-affinity inward rectifying channel AtAKT1 dominates $\mathrm{K}^{+}$uptake at intermediate concentrations $(1 \mathrm{mM})$. Under lower external $\mathrm{K}^{+}$concentrations $(100 \mu \mathrm{M})$, high-affinity potassium transporter AtHAK5, and together with AtAKT1, contributes to $\mathrm{K}^{+}$acquisition. AtHAK5 is the only transporter capable of taking up $\mathrm{K}^{+}$under extremely low concentrations $(<10 \mu \mathrm{M})$ [9]. Thus, it is imperative to characterize the function and expression pattern of high-affinity potassium transporters.

There are three families of $\mathrm{K}^{+}$transporters in plants: $\mathrm{HAK} / \mathrm{KUP} / \mathrm{KT}$ (High-affinity $\mathrm{K}^{+}$transporters/ $\mathrm{K}^{+}$uptake permeases $/ \mathrm{K}^{+}$transporters), HKT (High-affinity $\mathrm{K}^{+}$ transporters) and CPA (monovalent cation/proton antiporters) [10]. HKT transporters function as $\mathrm{K}^{+} / \mathrm{Na}^{+}$cotransporters or $\mathrm{Na}^{+}$transporters [11]. CPA family can be further divided into $\mathrm{NHX}\left(\mathrm{Na}^{+} / \mathrm{H}^{+}\right.$exchangers), $\mathrm{CHX}$ (Cation $/ \mathrm{H}^{+}$exchangers) and KEA ( $\mathrm{K}^{+}$efflux antiporters) subfamilies [10], and only several members (viz. AtCHX17, AtKEA4, AtKEA5 and AtKEA6) have been proved to function in $\mathrm{K}^{+}$transport $[12,13]$. The HAK/ $\mathrm{KUP} / \mathrm{KT}$ members in bacteria and fungi were named as KUP $\left(\mathrm{K}^{+}\right.$uptake permease) and HAK (High-affinity $\mathrm{K}^{+}$ transporter), respectively $[14,15]$. Diverse acronyms were assigned when the family members in plants were identified, viz. HAK for barley [16], KT ( $\mathrm{K}^{+}$transporter) or KUP for Arabidopsis [17-19], so composite names $\mathrm{HAK} / \mathrm{KUP} / \mathrm{KT}$ or $\mathrm{KT} / \mathrm{HAK} / \mathrm{KUP}$ or $\mathrm{KT} / \mathrm{KUP} / \mathrm{HAK}$ were widely used for the whole transporter family in plants [10, 20, 21]. HAK/KUP/KT (HAK hereafter) is the largest $\mathrm{K}^{+}$transporter family, and its members are predicted as $\mathrm{K}^{+} / \mathrm{H}^{+}$symporters [22]. HAK genes are absent in animal cells, but present in all known plant genomes [21], and first identified in barley [16] and Arabidopsis [17-19], and later also in rice [23, 24], wheat [25] and maize [26]. However, the mechanisms of HAK transporters in mediating $\mathrm{K}^{+}$uptake and transport are still completely unclear.

HAK transporters play the diverse roles in $\mathrm{K}^{+}$uptake and translocation, salt and drought stress response, as well as morphological development of root and shoot [21]. In Arabidopsis, AtHAK5 is a major $\mathrm{K}^{+}$deprivationinduced high-affinity $\mathrm{K}^{+}$uptake transporter, and is the only system being able to take up $\mathrm{K}^{+}$at external concentrations below $10 \mu \mathrm{M}[9,27]$. AtKUP7 is responsible for $\mathrm{K}^{+}$uptake at high $\mathrm{K}^{+}$concentrations, and may be also involved in $\mathrm{K}^{+}$transport into xylem sap, affecting $\mathrm{K}^{+}$translocation from roots to shoots [28]. In rice, the expression of OsHAK1 is up-regulated by $\mathrm{K}^{+}$deficiency in roots. Knockout of OsHAK1 reduces total $\mathrm{K}^{+}$uptake by approximately 80 and $65 \%$ at $0.05-0.1 \mathrm{mM}$ and $1 \mathrm{mM}$ of $\mathrm{K}^{+}$concentrations, respectively, while overexpression of OsHAK1 increases $\mathrm{K}^{+}$uptake and $\mathrm{K}^{+} / \mathrm{Na}^{+}$ratio [29]. OsHAK5 is expressed highly in root epidermis, stele and vascular tissues, and plays major roles not only in $\mathrm{K}^{+}$acquisition, but also in $\mathrm{K}^{+}$upward translocation from roots to shoots under low external $\mathrm{K}^{+}$conditions [22]. $\mathrm{K}^{+}$ homeostasis and $\mathrm{K}^{+} / \mathrm{Na}^{+}$balance are severely disturbed when plants are exposed to salt stress, as high $\mathrm{Na}^{+}$concentrations suppress the expression of $H A K$ genes at low $\mathrm{K}^{+}$concentrations [30,31]. However, AtHAK5 also plays a major role in maintaining high-affinity $\mathrm{K}^{+}$acquisition and plant growth under salt stress [31]. OsHAK1 is upregulated by salt stress under normal $\mathrm{K}^{+}$supply condition, but down-regulated at low $\mathrm{K}^{+}$concentrations [29]. The net $\mathrm{K}^{+}$uptake rate of oshak1 mutant was almost completely blocked by salt stress when $\mathrm{K}^{+}$concentration was below $0.05 \mathrm{mM}$, demonstrating that OsHAK1 plays pivotal role in enhancing salt tolerance in rice [29]. Overexpression of OsHAK5 also increases shoot $\mathrm{K}^{+} / \mathrm{Na}^{+}$ ratio and salt tolerance in rice [22]. Moreover, OsHAK1 knockout mutants reduce drought tolerance and growth at both vegetative and reproductive stages, while the over-expressed lines enhance drought tolerance and increase grain yield by $35 \%$ relative to wild type under drought condition [32]. In addition to $\mathrm{K}^{+}$uptake and translocation, and stress tolerance, some $H A K$ genes are also associated with tissue (root and shoot) morphology. It has been reported that AtHAK5 is the downstream target of ARF2 (Auxin response factor 2) and its overexpression results in longer primary roots under low $\mathrm{K}^{+}$ conditions [33]. AtKUP4/TRH1 (Tiny root hair 1) is required for polar localization of PIN1 (An auxin efflux transporter) in root apex, and for gravitropic responses, as well as normal initiation and formation of root hairs [34-37]. AtKUP2/SHY3 (Short hypocotyl 3) mutation affects cell expansion and leads to developmental defects in shoots [38]. In short, the versatile roles of $H A K$ genes have been recognized, including $\mathrm{K}^{+}$transport and abiotic stress response, but little has been known about the relevant molecular mechanisms.

Barley (Hordeum vulgare) ranks the fourth largest cereal crop worldwide with multiple use as animal feed, human food and brewing material [39]. In comparison with other cereal crops including rice, wheat and maize, barley is well known for its higher salt tolerance, which is largely attributed to its more $\mathrm{K}^{+}$uptake in roots, more $\mathrm{Na}^{+}$exclusion and vacuolar sequestration [40-42]. 
Although genome-wide identification of $H A K$ gene family has been accomplished in rice [24, 43], wheat [25], maize [26], cassava [44], peach [45], pear [46], and willow [47], the similar research has not been done in barley. The release of barley genome data [48] and recently improved annotated reference genome assembly [49] allow it possible to perform genome-wide identification of $H A K$ gene family in barley. In the present study, we identified 27 HAK genes (HvHAKs) from barley genome, and analyzed their phylogenetic relationships, conserved motifs and domains, gene structure, cis-acting elements, syntenic relationships, tissue expression patterns and expression profiling in responses to salt stress, osmotic stress and potassium deficiency.

\section{Results}

\section{Identification of HvHAKs in barley}

A total of $27 H v H A K$ genes were identified (Table 1; Additional file 1) using barley genome data [48] and recently improved annotated reference genome assembly [49]. The $27 \mathrm{H \nu HAK}$ genes were unevenly distributed on the seven chromosomes, with chromosome 2 containing eight genes, chromosomes 3 and 7 each containing four genes, chromosomes 1, 5 and 6 each containing three genes, and chromosome 4 containing two genes (Additional file 2). HAK genes in barley were named in the order of their locations on chromosomes (viz. HvHAK1 to $H v H A K 27$ ), and no tandem duplication event was observed (Table 1; Additional file 2). The length of HvHAK transporters varied from 724 to 875 amino acids (aa), and the number of transmembrane segments ranged from 10 to 14, with 11-12 (66.7\%) being the most abundant (Table 1). The isoelectric points (pI) of HvHAK proteins ranged from 5.65 to 8.99 , with only 6 members being below 7 (Table 1). The theoretical molecular weights (MW) of HvHAK proteins were in the range of 79.93 to $98.04 \mathrm{kDa}$. All the HvHAK transporters contained a typical "k_trans" domain (PF02705), which is specific to HAK potassium transporter family members (Table 1). In addition, all HvHAKs were localized on plasma membrane (Table 1).

\section{Phylogenetic analysis of HvHAK transporters}

The protein sequences of 13 AtKUPs in Arabidopsis [51], 27 OsHAKs in rice [24], 27 ZmHAKs in maize [26], and together with 27 HvHAKs in barley, were aligned with MAFFT (https://www.ebi.ac.uk/Tools/msa/ mafft/) and used to construct phylogenetic tree by maximum-likelihood (ML) method (Fig. 1). Based on the classification criteria of HAK transporters in Arabidopsis, rice and maize, the identified 27 barley HvHAKs could be classified into four clusters, and each of them could be further subdivided into sub-cluster A and B (Fig. 1). Clusters I and II each contained nine transporters, with IA, IB, IIA and IIB each containing two (HvHAK7 and 23), seven (HvHAK3, 8, 9, 12, 15, 17 and 21), four (HvHAK2, 5, 14 and 27) and five members (HvHAK4, 16, 22, 24 and 26), respectively (Fig. 1). Cluster III consisted of six transporters, with three members in both IIIA (HvHAK1, 10 and 20) and IIIB (HvHAK6, 11 and 18) (Fig. 1). Only three HvHAK transporters were categorized into cluster IV, with one member (HvHAK19) in IVA and two members (HvHAK13 and 25) in IVB (Fig. 1).

\section{Motif, domain and gene structure analyses of HvHAKs}

The conserved motifs and domains of HvHAK protein sequence and structures of $H \nu H A K$ genes were further analyzed (Fig. 2). Phylogenetic analysis confirmed the classification of 27 HvHAKs (Figs. 1 and 2a). Ten conserved motifs were identified in all 27 HvHAK sequences (Fig. 2b). According to NCBI conserved domain database, there are eight types of members for "K_trans superfamily" (cl15781), and three of them were identified in HvHAK protein sequences (Fig. 2b). The conserved domains of three, two and six HvHAK proteins were specifically identified as "K_trans", "PLN00151" and "kup", respectively, while those of the other 16 HvHAK sequences were identified as "K_trans superfamily" because of relatively low specificity (Fig. 2b). Cluster I and III contained "kup" and "K_trans superfamily", "PLN00151" and "K_trans superfamily", respectively; while cluster II and IV were solely comprised of "K trans superfamily" and "K_trans", respectively (Fig. 2b). Moreover, gene structures of barley HvHAKs were highly variable, containing 3-10 exons and 2-9 introns (Table 1; Fig. 2c).

Totally there were 14 (51.9\%) HvHAK genes which each contained eight introns, and there were 4, 3 and 2 genes which contained nine, six and seven introns, respectively; and other 4 genes possessed five, four, three and two introns, respectively (Fig. 2c). Intron numbers also differed among the different clusters (Fig. 2c); cluster I was the most variable, with six genes each containing two, three, four, six, eight and nine introns, respectively; three genes in cluster II had six, eight and nine introns, respectively; and two genes in both cluster III and IV had eight and nine, five and seven introns, respectively (Fig. 2c). These introns could be classified into three types based on phases: phase 0 , phase 1 and phase 2 [52]. Interestingly, phases 0 and 2 introns were detected, while phase 1 introns were not found in barley HvHAK genes (Fig. 2c). The phylogenetically most closely related members are prone to have the similar exon/intron structure. For instance, gene pairs $H v H A K 6$ and HvHAK11, HvHAK1 and HvHAK10,HvHAK5 and $H v H A K 14$, and HvHAK8 and HvHAK17 contained the same exon/intron numbers, respectively, and the exon 
Table 1 Twenty seven HvHAK genes identified in barley

\begin{tabular}{|c|c|c|c|c|c|c|c|c|c|c|}
\hline Name & Gene ID & $\begin{array}{l}\text { Length } \\
\text { (aa) }\end{array}$ & Intron & TMS & pl & $\begin{array}{l}\text { MW } \\
(\mathrm{kDa})\end{array}$ & $\begin{array}{l}\text { Conserved } \\
\text { domains }\end{array}$ & SL & $\begin{array}{l}\text { Alternative } \\
\text { names }\end{array}$ & $\begin{array}{l}\text { Reference for } \\
\text { alternative names }\end{array}$ \\
\hline HVHAK1 & HORVU.MOREX.r2.1 HG0007070.1 & 783 & 8 & 14 & 8.25 & 87.65 & $\begin{array}{l}\text { K_trans } \\
\text { superfamily }\end{array}$ & PM & & \\
\hline HVHAK2 & HORVU.MOREX.r2.1HG0054170.1 & 812 & 6 & 11 & 6.95 & 89.84 & $\begin{array}{l}\text { K_trans } \\
\text { superfamily }\end{array}$ & PM & & \\
\hline HvHAK3 & HORVU.MOREX.r2.1 HG0054790.1 & 736 & 4 & 11 & 8.58 & 80.97 & kup & PM & & \\
\hline HVHAK4 & HORVU.MOREX.r2.2HG0092260.1 & 780 & 6 & 11 & 8.25 & 86.84 & $\begin{array}{l}\text { K_trans } \\
\text { superfamily }\end{array}$ & PM & & \\
\hline HVHAK5 & HORVU.MOREX.r2.2HG0093910.1 & 777 & 8 & 13 & 8.78 & 86.71 & $\begin{array}{l}\text { K_trans } \\
\text { superfamily }\end{array}$ & PM & HVHAK2 & [16] \\
\hline HvHAKG & HORVU.MOREX.r2.2HG0117130.1 & 853 & 9 & 12 & 5.83 & 94.30 & PLN00151 & PM & & \\
\hline HVHAKT & HORVU.MOREX.r2.2HG0130150.1 & 875 & 2 & 12 & 8.87 & 98.04 & $\begin{array}{l}\text { K_trans } \\
\text { superfamily }\end{array}$ & PM & & \\
\hline HVHAK8 & HORVU.MOREX.r2.2HG0135750.1 & 775 & 8 & 12 & 8.95 & 86.41 & kup & PM & $\begin{array}{l}\text { HVHAK1; } \\
\text { HvHAKIA }\end{array}$ & {$[16,50]$} \\
\hline HVHAK9 & HORVU.MOREX.r2.2HG0135780.1 & 775 & 8 & 10 & 6.59 & 86.61 & kup & PM & & \\
\hline HVHAK10 & HORVU.MOREX.r2.2HG0158090.1 & 792 & 8 & 14 & 8.59 & 88.73 & $\begin{array}{l}\text { K_trans } \\
\text { superfamily }\end{array}$ & PM & & \\
\hline HVHAK11 & HORVU.MOREX.r2.2HG0158800.1 & 857 & 9 & 13 & 5.65 & 95.34 & $\begin{array}{l}\text { K_trans } \\
\text { superfamily }\end{array}$ & PM & & \\
\hline HVHAK12 & HORVU.MOREX.r2.3HG0261230.1 & 784 & 8 & 12 & 8.49 & 86.86 & $\begin{array}{l}\text { K_trans } \\
\text { superfamily }\end{array}$ & PM & & \\
\hline HVHAK13 & HORVU.MOREX.r2.3HG0261730.1 & 740 & 5 & 11 & 8.89 & 81.51 & K_trans & PM & & \\
\hline HVHAK14 & HORVU.MOREX.r2.3HG0262540.1 & 785 & 8 & 13 & 8.96 & 87.66 & $\begin{array}{l}\text { K_trans } \\
\text { superfamily }\end{array}$ & PM & HVHAK4 & {$[50]$} \\
\hline HVHAK15 & HORVU.MOREX.r2.3HG0265630.1 & 743 & 6 & 12 & 8.75 & 82.20 & kup & PM & & \\
\hline HVHAK16 & HORVU.MOREX.r2.4HG0314340.1 & 787 & 8 & 12 & 6.80 & 87.50 & $\begin{array}{l}\text { K_trans } \\
\text { superfamily }\end{array}$ & PM & & \\
\hline HVHAK17 & HORVU.MOREX.r2.4HG0339220.1 & 770 & 8 & 11 & 8.86 & 85.72 & kup & PM & HVHAK1B & {$[50]$} \\
\hline HVHAK18 & HORVU.MOREX.r2.5HG0395650.1 & 866 & 8 & 12 & 6.04 & 95.99 & PLN00151 & PM & HVHAK3 & {$[50]$} \\
\hline HVHAK19 & HORVU.MOREX.r2.5HG0402150.1 & 724 & 7 & 11 & 8.71 & 79.93 & K_trans & PM & & \\
\hline HVHAK2O & HORVU.MOREX.r2.5HG0420460.1 & 785 & 8 & 14 & 8.16 & 87.86 & $\begin{array}{l}\text { K_trans } \\
\text { superfamily }\end{array}$ & PM & & \\
\hline HVHAK21 & HORVU.MOREX.r2.6HG0455930.1 & 736 & 3 & 11 & 8.27 & 80.97 & kup & PM & & \\
\hline HVHAK22 & HORVU.MOREX.r2.6HG0507640.1 & 769 & 8 & 13 & 8.30 & 85.03 & $\begin{array}{l}\text { K_trans } \\
\text { superfamily }\end{array}$ & PM & & \\
\hline HVHAK23 & HORVU.MOREX.r2.6HG0508040.1 & 800 & 9 & 11 & 8.86 & 90.60 & $\begin{array}{l}\text { K_trans } \\
\text { superfamily }\end{array}$ & PM & & \\
\hline HvHAK24 & HORVU.MOREX.r2.7HG0557110.1 & 772 & 9 & 13 & 7.82 & 85.49 & $\begin{array}{l}\text { K_trans } \\
\text { superfamily }\end{array}$ & PM & & \\
\hline HVHAK25 & HORVU.MOREX.r2.7HG0564560.1 & 724 & 7 & 12 & 8.99 & 80.93 & K_trans & PM & & \\
\hline HVHAK26 & HORVU.MOREX.r2.7HG0599600.1 & 830 & 8 & 12 & 8.70 & 91.29 & $\begin{array}{l}\text { K_trans } \\
\text { superfamily }\end{array}$ & PM & & \\
\hline HVHAK27 & HORVU.MOREX.r2.7HG0610980.1 & 761 & 8 & 11 & 8.63 & 84.24 & $\begin{array}{l}\text { K_trans } \\
\text { superfamily }\end{array}$ & PM & & \\
\hline
\end{tabular}

aa Amino acids, $p /$ Isoelectric point, MW Molecular weight, SL Subcellular localization, TMS Transmembrane segment, PM Plasma membrane

configuration of each gene pair was almost the same regardless of the difference in intron length (Fig. 2c). However, the gene pairs of HvHAK4 and HvHAK16, and $H v H A K 7$ and $H v H A K 23$ showed the difference in both intron number and exon configuration (Fig. 2c).
Identification of cis-acting elements in HvHAK genes

A $2 \mathrm{~kb}$ sequence in the upstream of $H v H A K$ coding sequences was used for identification of cis-acting element. In total, 52 cis-acting elements were identified, and categorized into 6 types according to their functional 


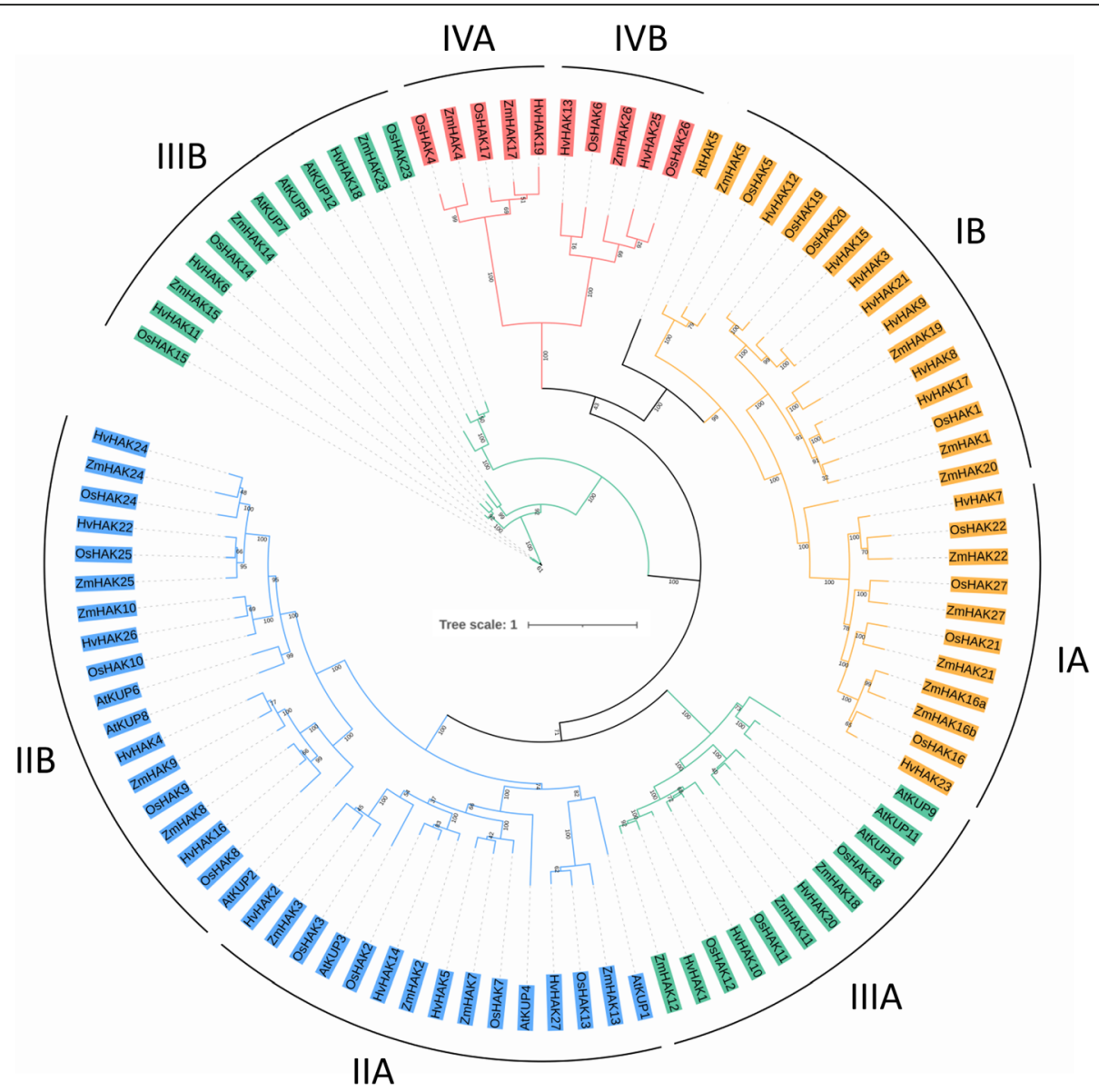

Fig. 1 Phylogenetic tree of HAK/KUP/KT family proteins from barley, rice, maize and Arabidopsis. The HAK/KUP/KT family proteins were divided into four clusters and were indicated with different colors. Yellow, blue, green and red clusters represented cluster I, II, III and IV, respectively. HV, Hordeum vulgare; Os, Oryza sativa; Zm, Zea mays; At, Arabidopsis thaliana

annotations (Fig. 3; Additional file 3). Light responsiveness-related elements were the most abundant (22, 42.3\%), followed by hormone response-related ( 9 , $17.3 \%)$, development/tissue specificity-related $(8,15.4 \%)$, promoter/enhancer element-related $(7,13.5 \%)$ and stressrelated (5, 9.6\%) ones (Fig. 3). Only one kind of elements was identified as circadian control (Fig. 3). CAAT-box and TATA-box from promoter/enhancer element, which are binding sites for RNA polymerase and responsible for transcription efficiency, were ubiquitously identified in all $H \nu H A K s$, indicating that they might play the pivotal roles in controlling the initiation and level of HvHAKs expression. Moreover, ABRE, as well as CGTCA-motif and TGACG-motif (from hormone response type), which are involved in abscisic acid (ABA) and methyl jasmonate (MeJA) responsiveness, respectively, were widely present in HvHAKs (Fig. 3). G-box in light responsiveness was also extensively distributed in promoter regions of $H v H A K s$ (Fig. 3). These results suggest that HvHAKs might play critical roles in phytohormone metabolism and environmental responses.

\section{Synteny analysis of HvHAKs in four monocotyledons}

The syntenic relationships of HvHAKs in four monocotyledonous plants (Triticum aestivum, Brachypodium distachyon, Oryza sativa and Zea mays) were investigated (Fig. 4; Additional file 4). Totally 73 pairs of orthologous genes were identified between barley and wheat, comprising 25 (92.6\%; except HvHAK3 and HvHAK9) HvHAKs and 72 TaHAKs (Fig. 4). Twenty-two pairs of orthologous genes were observed between barley and Brachypodium distachyon, barley and rice, and barley and maize, respectively, including 19 (70.4\%) HvHAKs and 21 BdHAKs, 17 (63.0\%) HvHAKs and 21 OsHAKs, $13(48.1 \%) H v H A K s$ and 20 ZmHAKs, respectively (Fig. 4). Orthologous genes of six barley HvHAKs (HvHAK1, 2, 15, 17, 21 and 23) were not detected in Brachypodium distachyon, rice and maize genomes, although found in wheat genome. Totally, $22 H v H A K s$ each had three orthologous genes in wheat, while only HvHAK24 had three orthologous genes in maize. Three HvHAKs, five HvHAKs and seven HvHAKs each had two orthologous genes in Brachypodium distachyon, rice and maize 


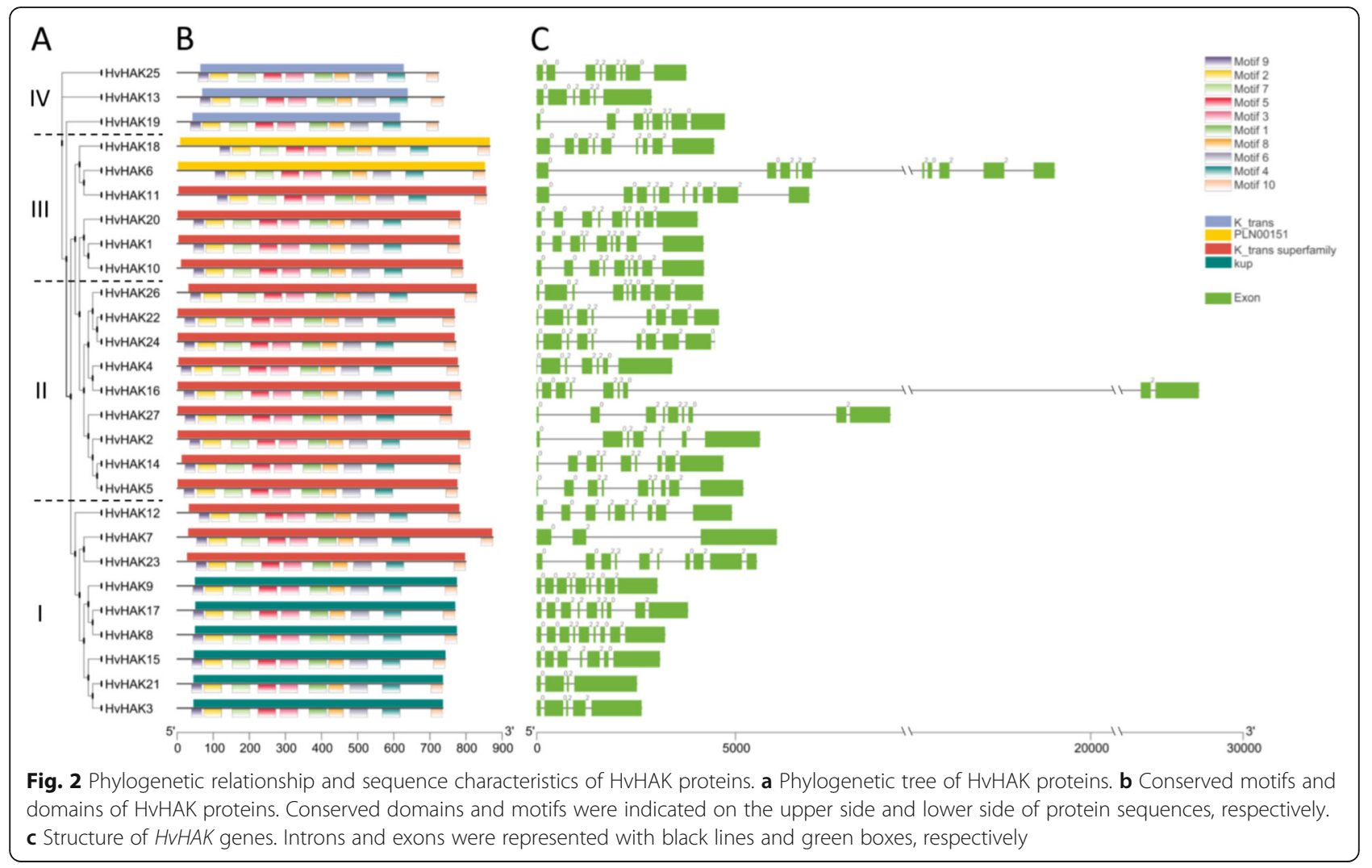

genomes, respectively. One TaHAK and one BdHAK were orthologous to two HvHAKs (HvHAK4 and $H v H A K 16)$, and one OsHAK and one ZmHAK were orthologous to the other two HvHAKs (HvHAK5 and $H v H A K 14)$. Besides, another ZmHAK was also orthologous to $H v H A K 12$ and $H v H A K 13$. These results indicate that $H v H A K s$ in barley are phylogenetically closer to TaHAKs in wheat than to BdHAKs in Brachypodium distachyon, OsHAKs in rice and ZmHAKs in maize.

\section{Tissue expression patterns of HvHAKs}

Tissue expression patterns of $H \nu H A K s$ were investigated in 15 tissues based on transcriptomic data from Barlex database (Additional file 5). Hierarchical cluster analysis revealed that $27 \mathrm{H \nu HAKs}$ could be divided into two groups according to their expression levels (Fig. 5). One group comprised $14 \mathrm{HvHAKs}$, and of them 13 genes were from cluster II and cluster III, with only one gene $(H v H A K 8)$ from cluster I (Fig. 5). Genes in this group were expressed ubiquitously in all 15 tissues with relatively high levels. The other group consisted of 13 genes, with eight from cluster I, two from cluster II and three genes from cluster IV (Fig. 5). The expression levels of $H v H A K s$ in this group were comparatively lower than that of the genes belonging to the former group, with eight genes being not detected in certain specific tissues (Fig. 5). Moreover, the expression of $H v H A K 2, H v H A K 3$ and $H v H A K 21$ was undetectable in seedling roots, while detected in the roots at $28 \mathrm{~d}$ after heading (Fig. 5). Furthermore, HvHAK13 was not expressed, and $H v H A K 15$ and $H v H A K 21$ were mildly expressed in developing grains (5 d after heading). However, $H v H A K 13$ was mildly expressed, but $H v H A K 15$ and $H v H A K 21$ were not expressed at $15 \mathrm{~d}$ after heading (Fig. 5). These results indicate that the expression of $H \nu H A K s$ is not only tissuedependent, but also varies with development phase.

\section{Expression patterns of $H v H A K s$ responding to abiotic stresses}

The effects of salt stress $(200 \mathrm{mM} \mathrm{NaCl})$, drought stress (20\% PEG8000) and potassium deficiency $\left(0.01 \mathrm{mM} \mathrm{K}^{+}\right)$ on barley growth were investigated. All three abiotic stress treatments inhibited shoot growth of barley seedlings, and resulted in abnormal root phenotypes (Additional file 6).

Under salt stress, all $H \nu H A K s$ examined were upregulated, but their expression patterns differed (Fig. 6). HvHAK5, HvHAK6, HvHAK18 and HvHAK27 were rapidly induced after one-hour salt treatment, and displayed continuous up-regulation, while $H v H A K 8$ was not induced until salt treatment for $3 \mathrm{~h}$ (Fig. 6a). Besides, $H \nu H A K 17$ was first down-regulated after treatment (1-6 $\mathrm{h})$, and was up-regulated after one-day salt treatment (Fig. 6a). In addition, HvHAK6, HvHAK18 and 


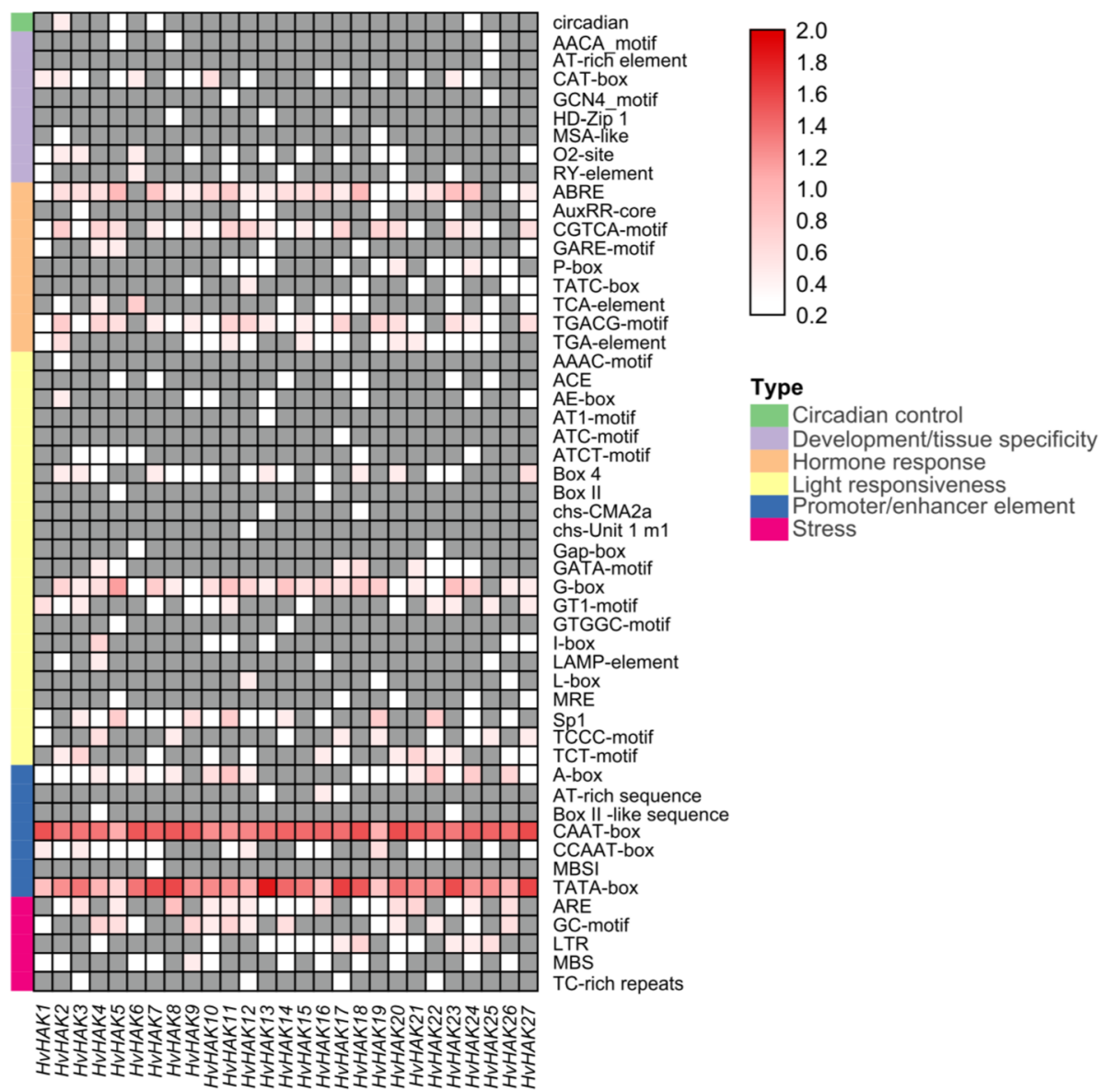

Fig. 3 Analysis of cis-acting elements in HvHAKs. Prediction of cis-acting elements was conducted on $2 \mathrm{~kb}$ sequences upstream of coding sequences of HVHAKs. Quantity of each kind of cis-acting elements were normalized by log ${ }_{10}($ number +1$)$ and then used for heatmap construction. All cis-acting elements could be divided into six types

HvHAK27 showed significantly higher up-regulation after long-term (3-6 d) salt treatment than after shortterm ( $1 \mathrm{~h}$ to $1 \mathrm{~d})$ treatment (Fig. 6a).

Under osmotic stress, HvHAK5, HvHAK6, and HvHAK27 were continuously induced during the whole experiment period (Fig. 6b). However, HvHAK8, HvHAK17 and HvHAK18 remained little change after one-hour treatment and even down-regulated after three-hour treatment, while they were up-regulated after treatment for $6 \mathrm{~h}$ and $1 \mathrm{~d}$ (Fig. 6b). Similarly, HvHAK5, HvHAK6 and HvHAK8 had significantly higher expression after long-term $(3 \mathrm{~d}$ and $6 \mathrm{~d})$ treatment than after short-term $(<1 \mathrm{~d})$ treatment (Fig. 6b).

All $H v H A K$ genes examined were up-regulated under $\mathrm{K}$ deficiency, except HvHAK6, which was downregulated at $1 \mathrm{~d}$ after treatment (Fig. 6c). Besides, the expression of $H v H A K 18$ remained relatively stable during the whole period of treatment (Fig. 6c).

\section{Discussion}

So far, the HAK/KUP/KT potassium transporter family has been identified and analyzed in many plant species, including Arabidopsis [50, 51], rice [23, 24, 43], wheat [25], maize [26] and cassava [44]. In the current study, we identified $27 H v H A K$ genes in barley (Table 1) using data of barley genome [48] and the improved annotated reference genome assembly [49], and then investigated their phylogenetic relationships, sequence characteristics, cis-acting elements, syntenic relationships, tissue expression patterns and expression profiling in response to abiotic stress.

\section{Nomenclature and classification of HvHAKs}

$H A K$ genes in maize and wheat were named according to their phylogenetic relationships with OsHAKs [25, 26]. The first identified $H A K$ gene in barley was named as HvHAK1 due to its high homology to Escherichia coli Kup and Schwanniomyces occidentalis HAK1, and another gene with only partial length (incomplete at $5^{\prime}$ end) was named as $H v H A K 2$ [16]. Rubio et al. [50] isolated three new cDNAs of $H A K$ genes in barley, and found one of them had more than $90 \%$ similarity to $H v H A K 1$. Consequently, they named it as $H v H A K 1 B$ 

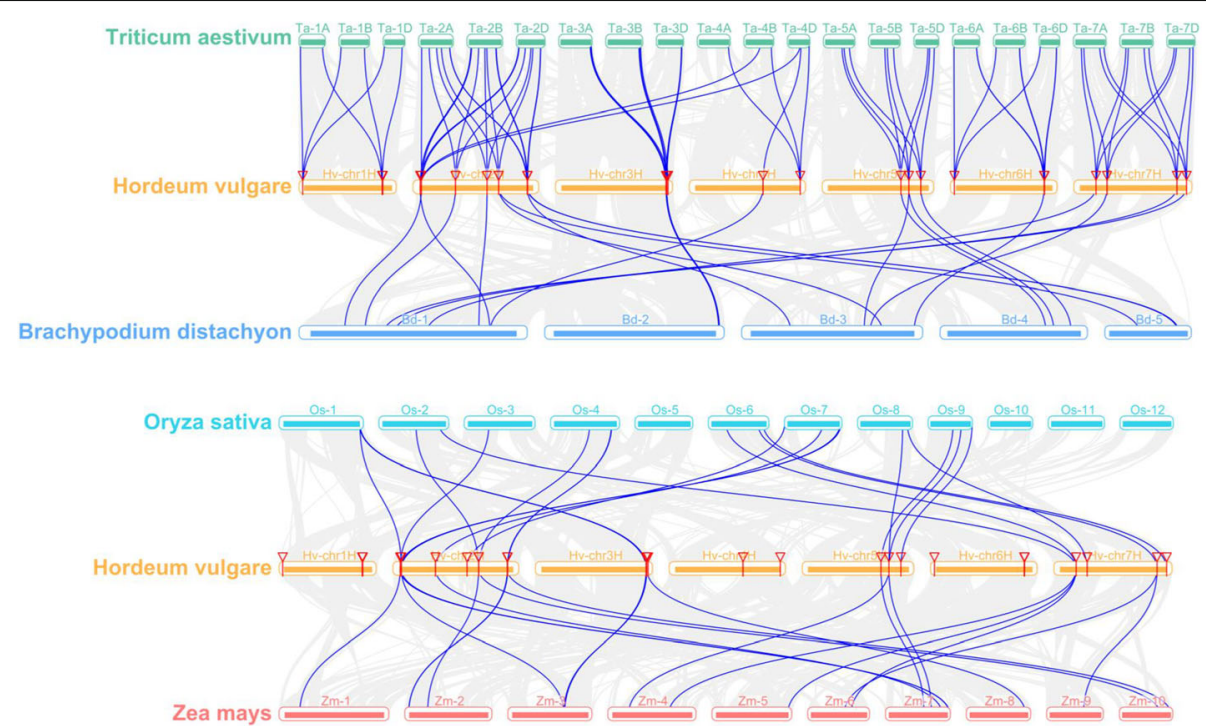

Fig. 4 Synteny analyses between HAK genes of barley and four monocotyledonous species (Triticum aestivum, Brachypodium distachyon, Oryza sativa and Zea mays). HvHAK genes are anchored on barley choromosomes. Grey lines indicated collinear blocks between barley and other plant genomes, while blue lines highlighted syntenic HAK gene pairs

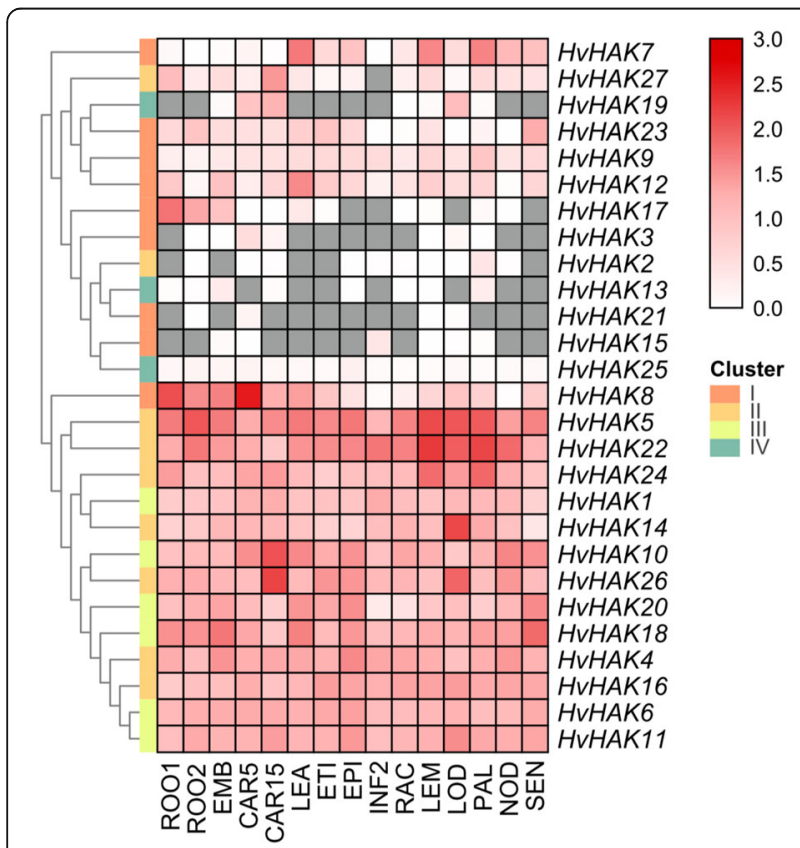

Fig. 5 Expression profiling of HVHAKs in 15 tissues based on transcriptomic data. FPKM values were normalized by $\log _{10}(\mathrm{FPKM}+1)$ transformation. ROO1, roots from seedlings ( $10 \mathrm{~cm}$ shoot stage); ROO2, roots (28 DAP); EMB, 4 day embryos; CAR5, developing grain (5 DAP); CAR15, developing grain (15 DAP); LEA, shoots from seedlings (10 cm shoot stage); ETI, etiolated seedling, dark condition (10 DAP); EPI, epidermal strips (28 DAP); INF, developing inflorescences (1-1.5 cm); RAC, inflorescences, rachis (35 DAP); LEM, inflorescences, lemma (42 DAP); LOD, inflorescences, lodicule (42 DAP); PAL, dissected inflorescences, palea (42 DAP); NOD, developing tillers, 3rd internode (42 DAP); SEN, senescing leaves (56 DAP)
( $H \nu H A K 1$ was designated as $H \nu H A K 1 A$ ), and the other two genes as $H \nu H A K 3$ and $H \nu H A K 4$, respectively. $H v H A K 1 / H v H A K 1 A$ and $H v H A K 1 B$ were phylogenetically most closely related to OsHAK1, while $H v H A K 2$, $H \nu H A K 3$ and $H v H A K 4$ showed the closest phylogenetic relationships with OsHAK7, OsHAK23 and OsHAK2, respectively (Table 1; Fig. 1). Therefore, naming HvHAKs based on their phylogenetic relationships with OsHAKs seems not to be straightforward and understandable. Thus, 27 HvHAKs were designated as HvHAK1 to HvHAK27 according to their chromosomal locations (Table 1).

OsHAKs were divided into four clusters on the basis of phylogenetic analysis, with cluster I, II, II and IV having eight, nine, six and four genes, respectively [24]. Barley $H A K$ genes can be also classified into four clusters according to the classification criteria of OsHAKs in rice, with cluster I, II, III and IV having nine, nine, six and three genes, respectively (Fig. 1). Notably, OsHAK13 was subdivided into cluster IIB previously [24], while it was subdivided into cluster IIA in this study (Fig. 1). This inconsistency might be caused by the difference in the methods of protein sequence alignment and phylogenetic construction. Gupta et al. [24] adopted ClustalX in the protein sequence alignment of OsHAKs, while MAFFT was used in this study, which was widely recommended for its higher accuracy in multiple sequence alignment [53]. In addition, neighbor joining (NJ) was used in the phylogenetic construction of OsHAKs [24]. NJ method is widely used due to its high computational efficiency, however, it cannot account for the high 
A
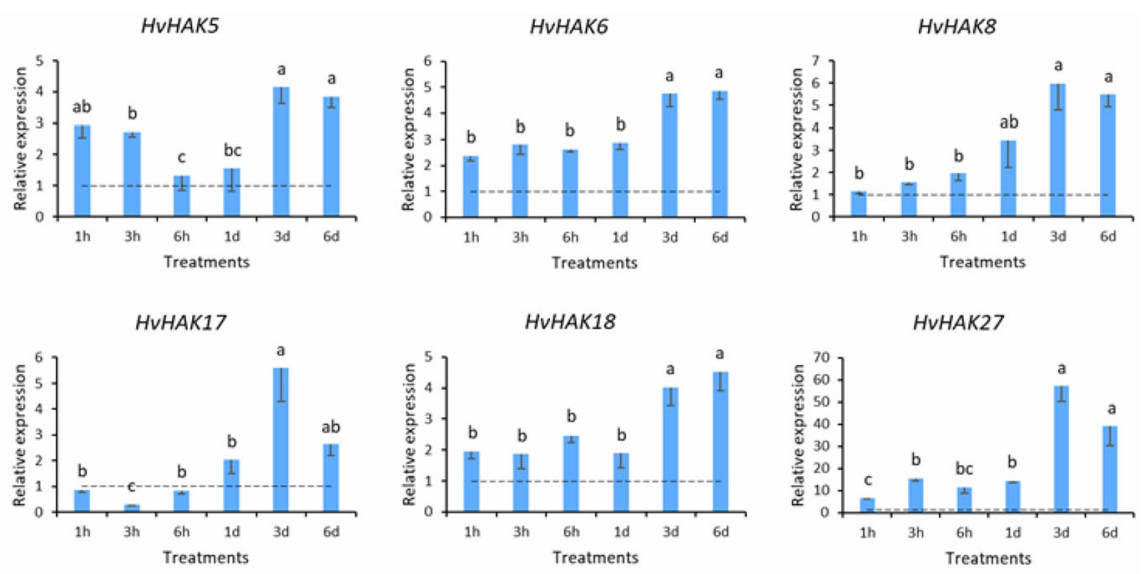

B
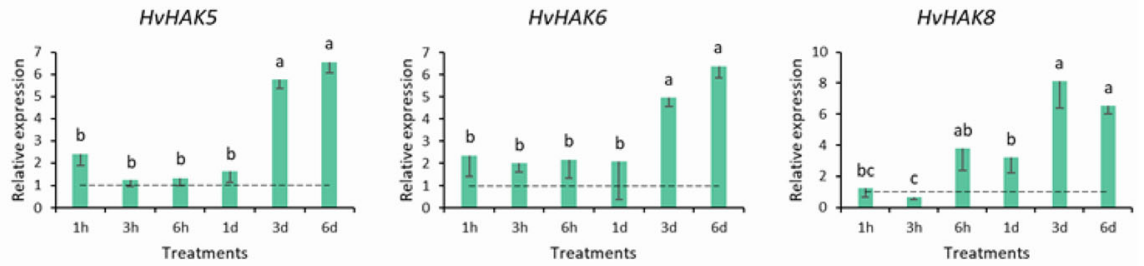

HVHAK17

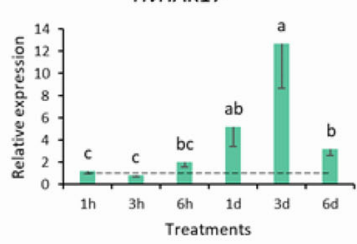

HVHAK18
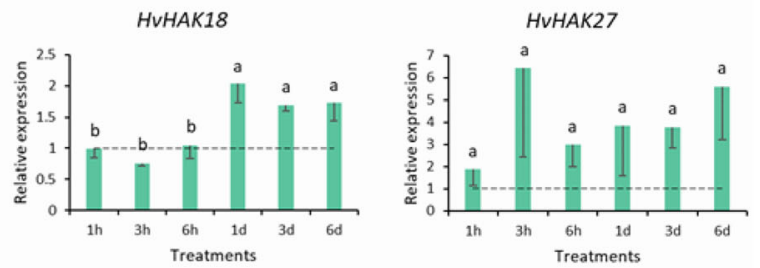

C

HvHAK5

HvHAK6
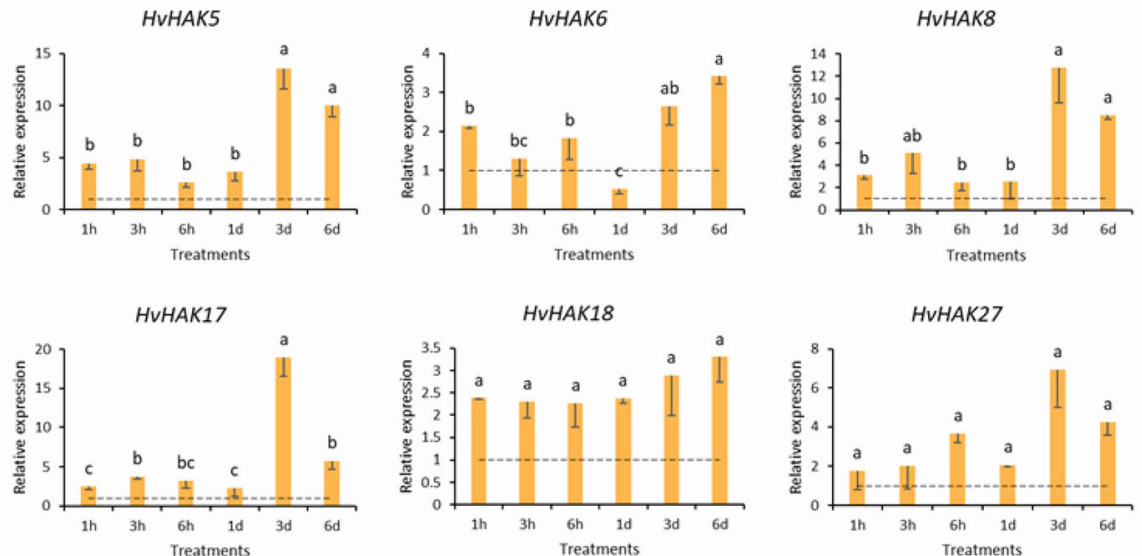

Fig. 6 Expression profiling of six HvHAK genes in response to abiotic stresses at seedling stage. a Expression of HvHAKs in response to salt stress (200 mM NaCl). b Expression of HvHAKs in response to osmotic stress (20\% PEG8000). c Expression of HvHAKs in response to potassium deficiency $\left(0.01 \mathrm{mM} \mathrm{K} \mathrm{K}^{+}\right.$. Dotted lines indicated the expression levels of HvHAKs in control seedlings. Lowercase letters indicated the significant difference at $p<0.05$

variances of large distance estimates and is also sensitive to the gaps in the sequence alignment [54]. Maximum likelihood, which was adopted in this study, has an obvious advantage over NJ method in understanding the sequence evolution process [54].
Similarities and differences of $H A K$ genes between barley and other plants

In this study, 27 HAK genes were identified in barley, basically similar to those found in rice [24], maize [26] and Brachypodium distachyon [55], although these 
plants show a huge difference in genome sizes, ranging from $\sim 355 \mathrm{Mb}$ of Brachypodium distachyon to $\sim 5.3 \mathrm{~Gb}$ of barley. In addition, the chromosome distribution of 27 HvHAK genes are uneven. Similarly, ZmHAKs were unevenly distributed on 10 chromosomes [26]. However, the chromosome distribution of OsHAKs are almost even [24]. The duplication events (including segmental duplication and tandem duplication) could be observed in both OsHAKs and ZmHAKs [26, 43], but not detected in HvHAKs (Additional file 2). The length in amino acid sequences of HvHAKs, OsHAKs and ZmHAKs were similar, ranging from 724 to 875,697 to 877 and 642 to 921 aa, respectively (Table 1) [24, 26]. Introns of $H v H A K$ genes varied from 2 to 9 , being similar to those of ZmHAK and OsHAK genes, with an exception of OsHAK22, which had only one intron. Interestingly, the $H A K$ genes with 8 introns were predominant in barley $(14,51.9 \%)$, rice $(12,44.4 \%)$ and maize $(16,59.3 \%)$ [24, 26]. Intron phase determines whether exons are targeted for alternative splicing. Generally, exons flanked by same-phase introns are possible for alternative splicing, while those flanked by the different-phase introns cannot be alternatively spliced [52]. On the whole the most introns of $H A K$ genes in rice and maize belonged to phase 0 and phase 1 , with only four and three introns in phase 2 for OsHAKs and ZmHAKs, respectively [24, 26]. However, introns of $H \nu H A K s$ all belong to phase 0 or phase 2 , and no introns were observed at phase 1 (Fig. 2), suggesting that $H v H A K s$ had the different expression over the phase from OsHAKs and ZmHAKs. The numbers of transmembrane segments (TMS) for OsHAKs, ZmHAKs and HvHAKs were in the range of 11-15, 9-14, and 1014 , respectively (Table 1$)[24,26]$. The HAK genes with 13 TMS $(11,40.7 \%)$ and 11 TMS (9, 33.3\%) were dominant in OsHAKs and ZmHAKs, while those with 11 and 12 TMS $(18,66.7 \%)$ were the most abundant in HvHAKs (Table 1) $[24,26]$.

All HAK proteins in rice, maize and wheat contain a conserved domain, "K_trans" (PF02705) [24-26]. In the present study, a conserved domain of "K_trans superfamily" (cl15781) was also identified in all HvHAK transporters (Fig. 2b). In addition, 1 conserved motifs were detected, and they were almost evenly distributed along HvHAK sequences, similar to motifs in TaHAKs [25]. For the cis-acting elements related to light responsiveness, they were the most abundant in HvHAKs, while quite fewer in OsHAKs and ZmHAKs [24, 26]. On the other hand, $\mathrm{Ca}^{2+}$-responsive elements could be detected in the most OsHAKs and ZmHAKs, but not observed in $H v H A K s$ (Fig. 3). It may be concluded that there is a great difference in cis-acting elements among OsHAKs, ZmHAKs and HvHAKs.

Based on the classification criteria of $H A K$ genes in rice and maize, $H v H A K s$ can be also divided into four clusters (Fig. 1). Synteny analysis identified 73 HAK orthologous gene pairs (comprising 25 HvHAKs and 72 TaHAKs) in barley and wheat genomes, much more than those between barley and Brachypodium distachyon genomes, and between rice and maize genomes, respectively (Fig. 4; Additional file 4). Interestingly, each of the 22 HvHAK genes was orthologous to three TaHAK genes, which might be attributed to the fact that wheat is an allohexaploid composed of three distinct ancestral genomes, viz. A, B and D [56]. If this explanation is reasonable, the phenomena that HvHAK15 and HvHAK16 were orthologous to two (lacking orthologous gene on genome D) and one TaHAKs (lacking orthologous genes on genomes A and B), respectively, can be described to gene loss events during evolution (Additional file 4). In addition, HvHAK4 was orthologous to four TaHAK genes on two chromosomes, suggesting that both gene loss and genomic recombination might have occurred (Additional file 4). Notably, according to the synteny analysis in this study, at least $72 H A K$ genes should be present in wheat genome, while only 56 TaHAK genes were identified in a previous research [25]. The inconsistency can be attributed to the imperfect assembly and annotation of wheat genome, as well as the workflow of gene family identification. Based on the barley genome assembly released in 2017 [48], only $24 H A K$ genes were identified in barley genome (three genes with incomplete $\mathrm{K}^{+}$transporter domains were arbitrarily discarded), while using the recently released barley reference genome assembly [49], we identified $27 H \nu H A K$ genes. On the other hand, 22 HAK orthologous gene pairs were identified between barley and other three plants examined in this study (Fig. 4; Additional file 4). In addition, 19, 17 and 13 HvHAKs were orthologous to 21 BdHAKs, 21 OsHAKs and $20 \mathrm{ZmHAKs}$, respectively, being consistent with the phylogenetic relationships between barley and these plants (Fig. 4; Additional file 4). No orthologous gene was found for $H \nu H A K 3$ and HvHAK9 in other three genomes, indicating that these two genes might originate from ectopic duplication of $H v H A K 2$ and $H v H A K 8$, respectively (Fig. 1) [57].

\section{Expression of HvHAKs in different tissues and in response to abiotic stresses}

Analysis of tissue expression pattern revealed that HvHAK genes could be divided into two groups (Fig. 5). One group, consisting of 14 genes, was constitutively and highly expressed in all 15 tissues, while another group, consisting of 13 genes, only expressed in the specific tissues with lower levels (Fig. 5). In the previous studies, expression of $H A K$ genes in Arabidopsis, rice and wheat also showed the great difference in the tissues and levels $[24,25,58]$. 
Salt, osmotic (drought) and K deficiency stresses had the dramatic effect on the expression of the examined six $H v H A K$ genes (Fig. 6). Maintaining efficient $\mathrm{K}^{+}$uptake is prerequisite for $\mathrm{K}^{+} / \mathrm{Na}^{+}$homeostasis and salt tolerance when plants are exposed to salt stress [59]. In this study, the expression of the six HvHAKs was upregulated under salt stress (Fig. 6a), contributing to high $\mathrm{K}^{+} / \mathrm{Na}^{+}$ratio and salt tolerance. The similar results were also observed for OsHAKs in rice and for MeKUPs in cassava $[44,60]$. It is well recognized that plants respond to drought stress via both ABA-dependent and ABAindependent pathways $[61,62]$. It was reported that increasing cytosolic ion concentrations through absorbing and accumulating $\mathrm{K}^{+}$is a relatively fast and costeffective way for plants to enhance intracellular osmotic adjustment and drought tolerance [63, 64]. In the present study, the expression of $H v H A K$ genes were upregulated in a time-dependent manner when plants were exposed to osmotic stress (drought) (Fig. 6b), being consistent with the previous reports on TaHAKs in wheat and MeKUPs in cassava $[25,44]$. In general, $\mathrm{K}^{+}$transporter genes are up-regulated under $\mathrm{K}$ deficiency conditions $[25,60,65]$. In this study, all examined $H v H A K$ genes were up-regulated under $\mathrm{K}$ deficiency treatment (Fig. 6c). It was reported that the expression of OsHAK1 was induced by $\mathrm{K}$ deficiency or salt stress, leading to increased $\mathrm{K}^{+}$uptake and $\mathrm{K}^{+} / \mathrm{Na}^{+}$ratio in roots [29]. In this study, the homolog genes of OsHAK1 in barley, HvHAK8 (alternative name as $H \nu H A K 1$ or $H \nu H A K 1 A$ ) and $H \nu H A K 17$ (alternative name as $H \nu H A K 1 B$ ), were also up-regulated under $\mathrm{K}$ deficiency or salt stress (Table 1; Figs. 1 and 6). In addition, the expression level of $H v H A K s$ varied dramatically with the time of stress exposure (Fig. 6). For example, HvHAK5 was slightly upregulated (1.2-2.4 folds) at $1 \mathrm{~h}$ to $1 \mathrm{~d}$ after osmotic stress, while it was up-regulated significantly (5.8-6.6 folds) at 3-6 d after the treatment (Fig. 6b). Moreover, three genes (HvHAK2, 3 and 21) were highly expressed in roots at $28 \mathrm{~d}$ after heading, but remained little change in seedling roots (Fig. 5). The same was true for HvHAK13, HvHAK15 and HvHAK21 in the developing grains (5 DAP and 15 DAP) (Fig. 5). Obviously, the response patterns of $H v H A K$ genes to abiotic stress are dual-phases or even multi-phases. Thus, the suitable time of sampling should be taken into consideration when expression patterns of these $H v H A K$ genes are analyzed and compared.

\section{Conclusions}

In the current study, $27 H A K$ genes (HvHAKs) were identified in barley. The expression of these $H v H A K s$ vary with the plant tissues and the time of exposed abiotic stress. Although all HvHAKs could be induced by salt, drought and $\mathrm{K}$ deficiency stresses, their response patterns and magnitudes showed the great variation. The obtained results should be helpful for us to make the comprehensive understanding of $H A K$ family in barley.

\section{Methods \\ Identification of HAK gene family in barley}

The amino acid sequences of $H A K / K U P / K T$ genes in Arabidopsis thaliana [51] and Oryza sativa [24] were downloaded from TAIR (https://www.arabidopsis.org/) and RAP-DB (https://rapdb.dna.affrc.go.jp/), respectively. The protein sequences of AtKUPs and OsHAKs were employed as queries to search against barley genome database (Morex V2 assembly) [49]. A total of 43 genes were identified as $H A K$ gene family candidates in barley genome. The putative $H v H A K s$ were then verified using NCBI Conserved Domain Database (https://www.ncbi. nlm.nih.gov/Structure/bwrpsb/bwrpsb.cgi), SMART (http://smart.embl-heidelberg.de/) and Pfam (https:// pfam.xfam.org/), and the sequences without complete potassium transporter domain were discarded. Finally, 27 genes were identified as $H A K$ gene family members in barley.

\section{Physicochemical properties, subcellular localizations and transmembrane segments of HvHAKs}

The theoretical molecular weights (MW) and isoelectric points (pI) of HvHAKs were calculated using ExPASy (https://web.expasy.org/compute_pi/). Subcellular localizations and trans-membrane segments of HvHAKs were predicted using BUSCA (http://busca.biocomp.unibo.it/) [66] and TMHMM (https://services.healthtech.dtu.dk/ service.php?TMHMM-2.0), respectively.

\section{Phylogenetic and syntenic analysis}

The protein sequences of AtKUPs [51], OsHAKs [24] and ZmHAKs [26] were downloaded from TAIR (https://www.arabidopsis.org/), RAP-DB (https://rapdb. dna.affrc.go.jp/index.html) and maizeGDB (https:// www.maizegdb.org/), respectively. HAK protein sequences from Arabidposis, rice, maize and barley were aligned using MAFFT (https://www.ebi.ac.uk/ Tools $/ \mathrm{msa} / \mathrm{mafft} /$ ) [67], and the phylogenetic tree was constructed with maximum-likelihood (ML) method using PhyML 3.0 (http://www.atgc-montpellier.fr/ phyml/) [68]. The genome sequences and annotations of wheat, Brachypodium distachyon, rice and maize were downloaded from EnsemblPlants (http://plants. ensembl.org/info/website/ftp/index.html), and their syntenic relations with barley were analyzed and visualized using TBtools [69]. 


\section{Sequence, chromosomal location and duplication} analyses of HvHAKs

Conserved motifs of HvHAKs were identified using MEME program (http://meme-suite.org/tools/meme) [70] with following parameters: classic motif discovery mode, zero or one occurrence per sequence (zoops), motif number was set to 10 . Conserved domains were analyzed using NCBI Conserved Domain Database (https://www.ncbi.nlm.nih.gov/Structure/bwrpsb/ bwrpsb.cgi). Motifs and conserved domains of HvHAKs as well as structures and chromosomal locations of HvHAKs were visualized using TBtools [69]. Gene duplication events were analyzed using TBtools following parameters described by Tombuloglu $[69,71]$.

\section{cis-acting element analysis}

The $2000 \mathrm{bp}$ sequences in the upstream of coding sequences of $H v H A K s$ were extracted for cis-acting element analysis using Plant CARE database (http:// bioinformatics.psb.ugent.be/webtools/plantcare/html/).

\section{Tissue expression patterns of HvHAKs}

Raw data (FPKM) were downloaded from BARLEX (https://apex.ipk-gatersleben.de/apex/f?p=284:46:::NO: RP:P46_GENE_CHOICE:3) and normalized by $\log _{10}(\mathrm{FPKM}+1)$ transform. Expression heatmap was drawn using TBtools [69].

\section{Plant materials, growth conditions and abiotic stress treatments}

Barley (Hordeum vulgare cv. Morex. From Professor Rugen Xu's lab, Yangzhou University) seeds were sterilized with $10 \%$ commercial $\mathrm{NaClO}$ for $15 \mathrm{~min}$ and rinsed with tap water for $30 \mathrm{~min}$ [64]. Sterilized seeds were germinated in basic salt medium (BSM, $0.5 \mathrm{mM} \mathrm{KCl}+0.1$ $\mathrm{mM} \mathrm{CaCl}{ }_{2}$ ) for $2 \mathrm{~d}$, and then BSM was changed to onefifth Hoagland solution for another $4 \mathrm{~d}$ in growth room with a photoperiod of $14 / 10 \mathrm{~h}$, light intensity of $200 \pm$ $25 \mu \mathrm{mol} \cdot \mathrm{m}^{-2} \cdot \mathrm{s}^{-1}$, temperature of $23 / 18^{\circ} \mathrm{C}$ (day/night) and relative humidity of $60 \%$. Barley seedlings were grown for $6 \mathrm{~d}$ and then subjected to salt stress $(200 \mathrm{mM}$ $\mathrm{NaCl}$ ) [72], osmotic stress (20\% PEG8000) [64] and potassium deficiency $\left(0.01 \mathrm{mM} \mathrm{K}^{+}\right)$[73] in background of 1/5 Hoagland solution. The seedlings growing in onefifth Hoagland solution were set as control. The solutions were renewed every 2 days. After treatments for 1 h, $3 \mathrm{~h}, 6 \mathrm{~h}, 1 \mathrm{~d}, 3 \mathrm{~d}$, and $6 \mathrm{~d}$, roots of barley seedlings under both control and abiotic stress conditions were sampled for RNA extraction and qRT-PCR. All samples were collected in three replicates.

\section{qRT-PCR}

Total RNA was extracted using MiniBEST Plant RNA Extraction Kit (9769, TaKaRa, Japan) following the manufacturer's instructions. The cDNA was synthesized from total RNA $(1 \mu \mathrm{g})$ using PrimeScript RT Master Mix (RR036A, TaKaRa, Japan) and was used as templates for qRT-PCR amplification. qRT-PCR amplification was performed with LightCycler 480 II (Roche, Basel, Switzerland) using iTaq Universal SYBR Green Supermix (1,725,124, Bio-Rad, USA). The relative gene expression was calculated based on the $2^{-\triangle \Delta C T}$ method using actin as the internal standard [74]. The primer sequences were listed in the Additional file 7.

\section{Abbreviations}

ARF2: Auxin response factor 2; CHX: Cation/ $\mathrm{H}^{+}$exchanger; CPA: Monovalent cation/proton antiporter; HAK: High-affinity $\mathrm{K}^{+}$transporter; HKT: High-affinity $\mathrm{K}^{+}$transporter; KEA: $\mathrm{K}^{+}$efflux antiporter; $\mathrm{KT}: \mathrm{K}^{+}$transporter; KUP: $\mathrm{K}^{+}$uptake

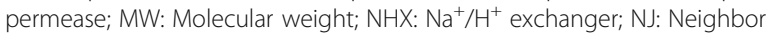
joining; NSCC: Non-selective cation channel; pl: Isoelectric point; SHY3: Short hypocotyl 3; TRH1: Tiny root hair 1

\section{Supplementary Information}

The online version contains supplementary material available at https://doi. org/10.1186/s12864-021-07633-y.

Additional file 1. Protein sequences of $27 \mathrm{HvHAKs.}$

Additional file 2 Chromosomal distribution of HVHAK genes.

Chromosome names are displayed on the left side of chromosomes. HvHAK gene names are indicated on the right side of chromosomes.

Additional file $\mathbf{3}$ cis-acting elements in HVHAK genes.

Additional file 4. Orthologous gene pairs in synteny analysis.

Additional file $\mathbf{5}$ Tissue expression patterns of HVHAKS.

Additional file 6 . Phenotypes of barley seedlings after abiotic stress treatments for $6 \mathrm{~d}$.

Additional file 7. Primers for qRT-PCR.

\section{Acknowledgements}

We thank PhD candidate Yuanyuan Wang from Zhejiang University for her comments and suggestions on phylogenetic analyses.

\section{Authors' contributions}

$K C$ and $G Z$ conceived the project. KC designed and conducted the experiments. KC performed the main data analysis. KC, FZ, JW and GZ contributed to result interpretation. KC wrote the manuscript. GZ revised the manuscript. All authors read and approved the final manuscript.

\section{Funding}

This study was supported by National Natural Science Foundation of China (31620103912, 31671678), China Agriculture Research System (CARS-05) and Jiangsu Collaborative Innovation Centre for Modern Crop Production (JCIC$\mathrm{MCP}$. The funders had no roles in study design, data collection and analysis, and result interpretation and manuscript writing.

\section{Availability of data and materials}

The datasets supporting the conclusions of this article are included within the article and its additional files.

\section{Declarations}

Ethics approval and consent to participate Not applicable.

Consent for publication

Not applicable. 


\section{Competing interests}

The authors declare that they have no competing interests.

\section{Author details}

'Institute of Crop and Nuclear Technology Utilization, Zhejiang Academy of Agricultural Sciences, Hangzhou 310021, China. ${ }^{2}$ Institute of Crop Science, Zhejiang University, Hangzhou 310058, China.

\section{Received: 25 October 2020 Accepted: 21 April 2021} Published online: 01 May 2021

\section{References}

1. Anschütz U, Becker D, Shabala S. Going beyond nutrition: regulation of potassium homoeostasis as a common denominator of plant adaptive responses to environment. J Plant Physiol. 2014;171(9):670-87. https://doi. org/10.1016/j.jplph.2014.01.009.

2. Wang $M$, Zheng $Q$, Shen $Q$, Guo $S$. The critical role of potassium in plant stress response. Int J Mol Sci. 2013;14(4):7370-90. https://doi.org/10.3390/ ijms14047370.

3. Hasanuzzaman M, Bhuyan MHMB, Nahar K, Hossain MS, Al Mahmud J, Hossen MS, et al. Potassium: a vital regulator of plant responses and tolerance to abiotic stresses. Agronomy. 2018;8(3). https://doi.org/10.3390/a gronomy8030031.

4. Fageria NK. The use of nutrients in crop plants. 1st ed. Boca Raton: CRC Press; 2009

5. Sardans J, Peñuelas J. Potassium: a neglected nutrient in global change. Glob Ecol Biogeogr. 2015;24(3):261-75. https://doi.org/10.1111/geb.12259.

6. Walker DJ, Leigh RA, Miller AJ. Potassium homeostasis in vacuolate plant cells. Proc Natl Acad Sci U S A. 1996;93(19):10510-4. https://doi.org/10.1073/ pnas.93.19.10510.

7. MacRobbie EAC. Control of volume and turgor in Stomatal guard cells. J Membr Biol. 2006;210(2):131-42. https://doi.org/10.1007/s00232-005-0851-7.

8. White PJ, Karley AJ. Potassium. In: Hell R, Mendel RR, editors. Cell Biology of Metals and Nutrients. Berlin: Springer Berlin Heidelberg; 2010. p. 199-224. https://doi.org/10.1007/978-3-642-10613-2_9.

9. Nieves-Cordones M, Alemán F, Martínez V, Rubio F. K+ uptake in plant roots. The systems involved, their regulation and parallels in other organisms. J Plant Physiol. 2014;171(9):688-95. https://doi.org/10.1016/j.jplph.2013.09.021.

10. Gierth $\mathrm{M}$, Mäser P. Potassium transporters in plants - involvement in $\mathrm{K}^{+}$ acquisition, redistribution and homeostasis. FEBS Lett. 2007;581(12):2348-56. https://doi.org/10.1016/j.febslet.2007.03.035.

11. Almeida P, Katschnig D, de Boer AH. HKT transporters--state of the art. Int J Mol Sci. 2013;14(10):20359-85. https://doi.org/10.3390/ijms141020359.

12. Cellier F, Conéjéro G, Ricaud L, Doan TL, Lepetit M, Gosti $F$, et al. Characterization of AtCHX17, a member of the cation/ $\mathrm{H}^{+}$exchangers, $\mathrm{CHX}$ family, from Arabidopsis thaliana suggests a role in $\mathrm{K}^{+}$homeostasis. Plant J. 2004;39(6):834-46. https://doi.org/10.1111/j.1365-313X.2004.02177.x.

13. Zhu X, Pan T, Zhang X, Fan L, Quintero FJ, Zhao H, et al. $K^{+}$efflux antiporters 4, 5, and 6 mediate $\mathrm{pH}$ and $\mathrm{K}^{+}$homeostasis in endomembrane compartments. Plant Physiol. 2018;178(4):1657-78. https://doi.org/10.1104/ pp.18.01053.

14. Schleyer M, Bakker EP. Nucleotide sequence and 3'-end deletion studies indicate that the $\mathrm{K}(+)$-uptake protein kup from Escherichia coli is composed of a hydrophobic core linked to a large and partially essential hydrophilic C terminus. J Bacteriol. 1993;175(21):6925-31. https://doi.org/10.1128/jb.175.21. 6925-6931.1993.

15. Bañuelos MA, Klein RD, Alexander-Bowman SJ, Rodríguez-Navarro A. A potassium transporter of the yeast Schwanniomyces occidentalis homologous to the Kup system of Escherichia coli has a high concentrative capacity. EMBO J. 1995;14(13):3021-7. https://pubmed.ncbi.nlm.nih.gov/7621 817. https://doi.org/10.1002/j.1460-2075.1995.tb07304.x.

16. Santa-María GE, Rubio F, Dubcovsky J, Rodríguez-Navarro A. The HAK1 gene of barley is a member of a large gene family and encodes a high-affinity potassium transporter. Plant Cell. 1997;9(12):2281-9. https://doi.org/10.1105/ tpc.9.12.2281.

17. Quintero FJ, Blatt MR. A new family of $\mathrm{K}^{+}$transporters from Arabidopsis that are conserved across phyla. FEBS Lett. 1997;415(2):206-11. https://doi.org/1 0.1016/S0014-5793(97)01125-3.

18. Fu HH, Luan S. AtKuP1: a dual-affinity $\mathrm{K}^{+}$transporter from Arabidopsis. Plant Cell. 1998;10(1):63-73. https://doi.org/10.1105/tpc.10.1.63.
19. Kim EJ, Kwak JM, Uozumi N, Schroeder JI. AtKUP1: an Arabidopsis gene encoding high-affinity potassium transport activity. Plant Cell. 1998;10(1):5162. https://doi.org/10.1105/tpc.10.1.51.

20. Grabov A. Plant KT/KUP/HAK potassium transporters: single family multiple functions. Ann Bot. 2007;99(6):1035-41. https://doi.org/10.1093/a ob/mcm066.

21. Li W, Xu G, Alli A, Yu L. Plant HAK/KUP/KT K K transporters: function and regulation. Semin Cell Dev Biol. 2018;74:133-41. https://doi.org/10.1016/j. semcdb.2017.07.009

22. Yang T, Zhang S, Hu Y, Wu F, Hu Q, Chen G, et al. The role of a potassium transporter OsHAK5 in potassium acquisition and transport from roots to shoots in rice at low potassium supply levels. Plant Physiol. 2014;166(2):94559. https://doi.org/10.1104/pp.114.246520.

23. Bañuelos MA, Garciadeblas B, Cubero B, Rodríguez-Navarro A. Inventory and functional characterization of the HAK potassium transporters of rice. Plant Physiol. 2002;130(2):784-95. https://doi.org/10.1104/pp.007781.

24. Gupta M, Qiu X, Wang L, Xie W, Zhang C, Xiong L, et al. KT/HAK/KUP potassium transporters gene family and their whole-life cycle expression profile in rice (Oryza sativa). Mol Gen Genomics. 2008;280(5):437-52. https:// doi.org/10.1007/s00438-008-0377-7.

25. Cheng X, Liu X, Mao W, Zhang X, Chen S, Zhan K, et al. Genome-wide identification and analysis of HAK/KUP/KT potassium transporters gene family in wheat (Triticum aestivum L.). Int J Mol Sci. 2018;19(12). https://doi. org/10.3390/ijms19123969.

26. Zhang Z, Zhang J, Chen Y, Li R, Wang H, Wei J. Genome-wide analysis and identification of HAK potassium transporter gene family in maize (Zea mays L.). Mol Biol Rep. 2012;39(8):8465-73. https://doi.org/10.1007/s11033-012-1 700-2.

27. Gierth M. The potassium transporter AtHAK5 functions in $\mathrm{K}^{+}$deprivationinduced high-affinity $\mathrm{K}^{+}$uptake and $A K T 1 \mathrm{~K}^{+}$channel contribution to $\mathrm{K}^{+}$ uptake kinetics in Arabidopsis roots. Plant Physiol. 2005;137(3):1105-14. https://doi.org/10.1104/pp.104.057216.

28. Han M, Wu W, Wu WH, Wang Y. Potassium transporter KUP7 is involved in $\mathrm{K}^{+}$acquisition and translocation in Arabidopsis root under $\mathrm{K}^{+}$-limited conditions. Mol Plant. 2016;9(3):437-46. https://doi.org/10.1016/j.molp.2016. 01.012.

29. Chen G, Hu Q, Luo L, Yang T, Zhang S, Hu Y, et al. Rice potassium transporter OsHAK1 is essential for maintaining potassium-mediated growth and functions in salt tolerance over low and high potassium concentration ranges. Plant Cell Environ. 2015;38(12):2747-65. https://doi.org/10.1111/ pce.12585.

30. Nieves-Cordones M, Miller AJ, Alemán F, Martínez V, Rubio F. A putative role for the plasma membrane potential in the control of the expression of the gene encoding the tomato high-affinity potassium transporter HAK5. Plant Mol Biol. 2008;68(6):521-32. https://doi.org/10.1007/s11103-008-9388-3.

31. Nieves-Cordones M, Alemán F, Martínez V, Rubio F. The Arabidopsis thaliana HAK5 $\mathrm{K}^{+}$transporter is required for plant growth and $\mathrm{K}^{+}$acquisition from low $\mathrm{K}^{+}$solutions under saline conditions. Mol Plant. 2010;3(2):326-33. https://doi.org/10.1093/mp/ssp102.

32. Chen G, Liu C, Gao Z, Zhang Y, Jiang H, Zhu L, et al. OsHAK1, a high-affinity potassium transporter, positively regulates responses to drought stress in rice. Front Plant Sci. 2017;8:1885.

33. Zhao S, Zhang ML, Ma TL, Wang Y. Phosphorylation of ARF2 relieves its repression of transcription of the $\mathrm{K}^{+}$transporter gene HAK5 in response to low potassium stress. Plant Cell. 2016;28(12):3005-19. https://doi.org/10.11 05/tpc.16.00684.

34. Rigas S, Ditengou FA, Ljung K, Daras G, Tietz O, Palme K, et al. Root gravitropism and root hair development constitute coupled developmental responses regulated by auxin homeostasis in the Arabidopsis root apex. New Phytol. 2013;197(4):1130-41. https://doi.org/10.1111/nph.12092.

35. Daras G, Rigas S, Tsitsekian D, lacovides TA, Hatzopoulos P. Potassium transporter TRH1 subunits assemble regulating root-hair elongation autonomously from the cell fate determination pathway. Plant Sci. 2015;231: 131-7. https://doi.org/10.1016/j.plantsci.2014.11.017.

36. Rigas S, Debrosses G, Haralampidis K, Vicente-Agullo F, Feldmann KA, Grabov A, et al. TRH1 encodes a potassium transporter required for tip growth in Arabidopsis root hairs. Plant Cell. 2001;13(1):139-51. https://doi. org/10.1105/tpc.13.1.139.

37. Desbrosses G, Josefsson C, Rigas S, Hatzopoulos P, Dolan L. AKT1 and TRH1 are required during root hair elongation in Arabidopsis. J Exp Bot. 2003; 54(383):781-8. https://doi.org/10.1093/jxb/erg066. 
38. Elumalai RP, Nagpal P, Reed JW. A mutation in the Arabidopsis KT2/KUP2 potassium transporter gene affects shoot cell expansion. Plant Cell. 2002; 14(1):119-31. https://doi.org/10.1105/tpc.010322.

39. Cai K, Chen X, Han Z, Wu X, Zhang S, Li Q, et al. Screening of worldwide barley collection for drought tolerance: the assessment of various physiological measures as the selection criteria. Front Plant Sci. 2020;11: 1159. https://doi.org/10.3389/fpls.2020.01159.

40. Munns R, Tester M. Mechanisms of salinity tolerance. Annu Rev Plant Biol. 2008;59(1):651-81. https://doi.org/10.1146/annurev.arplant.59.032607.092911.

41. Chen Z, Newman I, Zhou M, Mendham N, Zhang G, Shabala S. Screening plants for salt tolerance by measuring $\mathrm{K}^{+}$flux: a case study for barley. Plant Cell Environ. 2005;28(10):1230-46. https://doi.org/10.1111/j.1365-3040.2005. 01364.x.

42. Wu H. Plant salt tolerance and $\mathrm{Na}^{+}$sensing and transport. Crop J. 2018;6(3): 215-25. https://doi.org/10.1016/j.cj.2018.01.003.

43. Yang Z, Gao Q, Sun C, Li W, Gu S, Xu C. Molecular evolution and functional divergence of HAK potassium transporter gene family in rice (Oryza sativa $\mathrm{L}$. ). J Genet Genom. 2009;36(3):161-72. https://doi.org/10.1016/\$1673-852 7(08)60103-4.

44. Ou W, Mao X, Huang C, Tie W, Yan Y, Ding Z, et al. Genome-wide identification and expression analysis of the KUP family under abiotic stress in cassava (Manihot esculenta Crantz). Front Physiol. 2018;9:17. https://doi. org/10.3389/fphys.2018.00017.

45. Song ZZ, Ma RJ, Yu ML. Genome-wide analysis and identification of KT/ HAK/KUP potassium transporter gene family in peach (Prunus persica). Genet Mol Res. 2015;14(1):774-87. https://doi.org/10.4238/2015.January.3 0.21 .

46. Li Y, Peng L, Xie C, Shi X, Dong C, Shen Q, et al. Genome-wide identification, characterization, and expression analyses of the HAK/KUP/KT potassium transporter gene family reveals their involvement in $\mathrm{K}^{+}$deficient and abiotic stress responses in pear rootstock seedlings. Plant Growth Regul. 2018;85(2):187-98. https://doi.org/10.1007/s10725-018-0382-8.

47. Liang M, Liang M, Gao Y, Gao Y, Mao T, Mao T, et al. Characterization and expression of KT/HAK/KUP transporter family genes in willow under potassium deficiency, drought, and salt stresses. Biomed Res Int. 2020;2020: 2690760.

48. Mascher M, Gundlach H, Himmelbach A, Beier S, Twardziok SO, Wicker T, et al. A chromosome conformation capture ordered sequence of the barley genome. Nature. 2017;544(7651):427-33. https://doi.org/10.1038/nature22 043.

49. Monat C, Padmarasu S, Lux T, Wicker T, Gundlach H, Himmelbach A, et al. TRITEX: chromosome-scale sequence assembly of Triticeae genomes with open-source tools. Genome Biol. 2019;20:1-18.

50. Rubio F, Guillermo SM, Rodríguez-Navarro A. Cloning of Arabidopsis and barley cDNAs encoding HAK potassium transporters in root and shoot cells. Physiol Plant. 2000;109(1):34-43. https://doi.org/10.1034/j.1399-3054.2000.1 00106.x.

51. Mäser P, Thomine S, Schroeder Jl, Ward JM, Hirschi K, Sze H, et al. Phylogenetic relationships within cation transporter families of Arabidopsis. Plant Physiol. 2001;126(4):1646-67. https://doi.org/10.1104/pp.126.4.1646.

52. Choudhuri S. Chapter 1 - Fundamentals of Genes and Genomes. In: Choudhuri SBT-B for B, editor. Bioinformatics for Beginners. Oxford: Academic; 2014. p. 1-25. https://doi.org/10.1016/B978-0-12-410471-6. 00001-3.

53. Pais FS, de Cássia Ruy P, Oliveira G, Coimbra RS. Assessing the efficiency of multiple sequence alignment programs. Algorithms Mol Biol. 2014;9(1):4. https://doi.org/10.1186/1748-7188-9-4.

54. Yang Z, Rannala B. Molecular phylogenetics: principles and practice. Nat Rev Genet. 2012;13(5):303-14. https://doi.org/10.1038/nrg3186.

55. Nieves-Cordones M, Ródenas R, Chavanieu A, Rivero RM, Martinez V, Gaillard I, et al. Uneven HAK/KUP/KT protein diversity among angiosperms: species distribution and perspectives. Front Plant Sci. 2016;7:127.

56. Winfield MO, Wilkinson PA, Allen AM, Barker GLA, Coghill JA, Burridge A, et al. Targeted re-sequencing of the allohexaploid wheat exome. Plant Biotechnol J. 2012;10(6):733-42. https://doi.org/10.1111/j.1467-7652.2012. 00713.x.

57. Leister D. Tandem and segmental gene duplication and recombination in the evolution of plant disease resistance genes. Trends Genet. 2004:20(3): 116-22. https://doi.org/10.1016/j.tig.2004.01.007.
58. Ahn SJ, Shin R, Schachtman DP. Expression of KT/KUP genes in Arabidopsis and the role of root hairs in $\mathrm{K}^{+}$uptake. Plant Physiol. 2004;134(3):1135-45. https://doi.org/10.1104/pp.103.034660.

59. Cuin TA, Bose J, Stefano G, Jha D, Tester M, Mancuso S, et al. Assessing the role of root plasma membrane and tonoplast $\mathrm{Na}^{+} / \mathrm{H}^{+}$exchangers in salinity tolerance in wheat: in planta quantification methods. Plant Cell Environ. 2011;34(6):947-61. https://doi.org/10.1111/j.1365-3040.2011.02296.x.

60. Okada T, Nakayama H, Shinmyo A, Yoshida K. Expression of OsHAK genes encoding potassium ion transporters in rice. Plant Biotechnol. 2008;25(3): 241-5. https://doi.org/10.5511/plantbiotechnology.25.241.

61. Akpinar BA, Avsar B, Lucas SJ, Budak H. Plant abiotic stress signaling. Plant Signal Behav. 2012;7(11):1450-5. https://doi.org/10.4161/psb.21894.

62. Lucas S, Durmaz E, Akpnar BA, Budak H. The drought response displayed by a DRE-binding protein from Triticum dicoccoides. Plant Physiol Biochem. 2011:49(3):346-51. https://doi.org/10.1016/j.plaphy.2011.01.016.

63. Shabala S, Demidchik V, Shabala L, Cuin TA, Smith SJ, Miller AJ, et al. Extracellular $\mathrm{Ca}^{2+}$ ameliorates $\mathrm{NaCl}$-induced $\mathrm{K}^{+}$loss from Arabidopsis root and leaf cells by controlling plasma membrane $\mathrm{K}^{+}$-permeable channels. Plant Physiol. 2006;141(4):1653-65. https://doi.org/10.1104/pp.106.082388.

64. Cai K, Gao H, Wu X, Zhang S, Han Z, Chen X, et al. The ability to regulate transmembrane potassium transport in root is critical for drought tolerance in barley. Int J Mol Sci. 2019;20(17):4111. https://doi.org/10.3390/ijms201 74111.

65. Wang Y, Wu WH. Regulation of potassium transport and signaling in plants. Curr Opin Plant Biol. 2017;39:123-8. https://doi.org/10.1016/j.pbi.2017.06.006.

66. Savojardo C, Martelli PL, Fariselli P, Profiti G, Casadio R. BUSCA: an integrative web server to predict subcellular localization of proteins. Nucleic Acids Res. 2018;46(W1):W459-66. https://doi.org/10.1093/nar/gky320.

67. Madeira F, Park YM, Lee J, Buso N, Gur T, Madhusoodanan N, et al. The EMBL-EBI search and sequence analysis tools APIs in 2019. Nucleic Acids Res. 2019;47(W1):W636-41. https://doi.org/10.1093/nar/gkz268.

68. Guindon S, Dufayard J-F, Lefort V, Anisimova M, Hordijk W, Gascuel O. New algorithms and methods to estimate maximum-likelihood phylogenies: assessing the performance of PhyML 3.0. Syst Biol. 2010;59(3):307-21. https://doi.org/10.1093/sysbio/syq010.

69. Chen C, Chen H, Zhang Y, Thomas HR, Frank MH, He Y, et al. TBtools: an integrative toolkit developed for interactive analyses of big biological data. Mol Plant. 2020;13(8):1194-202. https://doi.org/10.1016/j.molp.2020.06.009.

70. Bailey TL, Boden M, Buske FA, Frith M, Grant CE, Clementi L, et al. MEME SUITE: tools for motif discovery and searching. Nucleic Acids Res. 2009; 37(Web Server):W202-8. https://doi.org/10.1093/nar/gkp335.

71. Tombuloglu H. Genome-wide analysis of the auxin response factors (ARF) gene family in barley (Hordeum vulgare L.). J Plant Biochem Biotechnol. 2019;28(1):14-24. https://doi.org/10.1007/s13562-018-0458-6.

72. Shen Q, Fu L, Dai F, Jiang L, Zhang G, Wu D. Multi-omics analysis reveals molecular mechanisms of shoot adaption to salt stress in Tibetan wild barley. BMC Genomics. 2016;17(1):1-15. https://doi.org/10.1186/s12864-01 6-3242-9.

73. Ye Z, Zeng J, Li X, Zeng F, Zhang G. Physiological characterizations of three barley genotypes in response to low potassium stress. Acta Physiol Plant. 2017;39(1):1-10. https://doi.org/10.1007/s11738-016-2300-x.

74. Livak KJ, Schmittgen TD. Analysis of relative gene expression data using real-time quantitative PCR and the $2^{-\Delta \Delta C T}$ method. Methods. 2001;25(4):4028. https://doi.org/10.1006/meth.2001.1262.

\section{Publisher's Note}

Springer Nature remains neutral with regard to jurisdictional claims in published maps and institutional affiliations. 